\title{
Medical Image Segmentation Based on Edge Detection Techniques
}

\author{
${ }^{1}$ Bnar M. Ghafour, ${ }^{2}$ Nassir H. Salman and ${ }^{3}$ Gullanar M. Hadi \\ ${ }^{1,3}$ Department of Software Engineering - Salahaddin University, Erbil, Iraq \\ ${ }^{2}$ Department of Computer Science, Cihan Univ, Erbil, Iraq \\ bnar_e@yahoo.com; nahu64@yahoo.com; gullanarm@yahoo.com
}

\begin{abstract}
In this article a new combination of image segmentation techniques including K-means clustering, watershed transform, region merging and growing algorithm was proposed to segment computed tomography(CT) and magnetic resonance(MR) medical images.

The first stage in the proposed system is "preprocessing" for required image enhancement, cropped, and convert the images into .mat or png ...etc image file formats then the image will be segmented using combination methods (clustering, region growing, and watershed, thresholding). Some initial over-segmentation appears due to the high sensitivity of the watershed algorithm to the gradient image intensity variations. Here, $\mathrm{K}$ - means and region growing with correct thresholding value are used to overcome that over segmentations. In our system the number of pixels of segmented area is calculated which is very important for medical image analysis for diseases or medicine effects on affected area of human body also displaying the edge map.

The results show that using clustering method output to region growing as input image, gives accurate and very good results compare with watershed technique which depends on gradient of input image, the mean and the threshold values which are chosen manually. Also the results show that the manual selection of the threshold value for the watershed is not as good as automatically selecting, where data misses may be happen.
\end{abstract}

Keywords: Medical image segmentation, K-means Clustering, Watershed, Region growing and merging, $\mathrm{CT}$ and $\mathrm{MR}$ images

\section{Introduction}

Medical imaging is an essential part of modern healthcare. Medical imaging technologists take X-rays, mammograms, ultrasounds and computed tomography images to help diagnose patients' injuries and diseases. on another hand, medical imaging (diagnostic radiography) is the practice of taking medical images of patients' internal body parts. A range of technologies are used, including radiography, fluoroscopy, angiography, computed tomography, mammography, ultrasound, magnetic resonance imaging and nuclear medicine.

Medical imaging professionals are a vital part of a healthcare team as the images they collect are usually used to confirm or exclude a medical diagnosis, to advise on the treatment of illness, monitor patient progress, or provide medical screening information for doctors and other medical specialists. Finally and as a conclusion; Medical imaging is the technique, process and art of creating visual representations of the interior of a body for clinical analysis and medical intervention. Medical imaging seeks to reveal internal structures hidden by the skin and bones, as well as to diagnose and 
Bnar M. Ghafour, Nassir H. Salman and Gullanar M. Hadi; Medical Image Segmentation Based on Edge Detection Techniques. Advances in I mage and Video Processing, Volume 3 No 2, April (2015); pp: 1-9

treat disease. Medical imaging also establishes a database of normal anatomy and physiology to make it possible to identify abnormalities. Although imaging of removed organs and tissues can be performed for medical reasons, such procedures are usually considered part of pathology instead of medical imaging.

\section{Segmentation of medical images}

Segmentation is the process dividing an image into regions with similar properties such as gray level, color, texture, brightness, and contrast.[1] and [2 - 4]. The role of segmentation is to subdivide the objects in an image; in case of medical image segmentation the aim is to:

- $\quad$ Study anatomical structure, Diagnosis

- Identify Region of Interest i.e. locate tumor, lesion and other abnormalities

- Measure tissue volume to measure growth of tumor (also decrease in size of tumor with treatment)

- Help in treatment planning prior to radiation therapy; in radiation dose calculation

- $\quad$ Surgery planning

\section{Methods available for MR image segmentation:}

MR imaging is specifically used in brain imaging and thus lot of research work has been done particularly in the areas of MR brain image segmentation [5-8]. The main goal in brain MR segmentation is to segment gray matter, white matter and cerebrospinal fluid. Segmentation is also used to find out the regions corresponding to lesions tumors, cyst, edema, and other pathologies and for this mostly T1- weighted images are used.

Most of the segmentation methods available for $C T$ and MR images segmentation are intensity based i.e. gray level based hence; the segmentation results are affected by (1) intensity inhomogeneities and (2) partial volume effects. Accordingly, different researchers have proposed methods for correction of these problems. For more details see [1].

\subsection{Region growing method}

Region growing is a bottom-up procedure that starts with a set of seed pixels. The aim is to grow a uniform connected region from each seed. Segmentation by growing a region from seed point using intensity mean measure. The region is iteratively grown by comparing all unallocated neighbouring pixels to the region. The difference between a pixel's intensity value and the region's mean, is used as a measure of similarity. The pixel with the smallest difference measured this way is allocated to the region. This process stops when the intensity difference between region mean and new pixel becomes larger than a certain threshold. Starting from the seed the intensity values of each pixel is compared with its neighbors and if it is within the threshold, it'll be marked as one.A pixel is added to a region if and only if:

- It has not been assigned to any other region.

- It is a neighbor of that region.

- The new region created by addition of the pixel is still uniform.

[9]*the region growing method we used after modify is proposed by Danial kellner Mathwork: Recursive region growing algorithm for 2D/3D grayscale images with polygon and binary mask output (2011). 


\subsection{Limitations of region growing}

Note that a complete segmentation of an image must satisfy a number of criteria:

- $\quad$ All pixels must be assigned to regions

- $\quad$ Each pixel must belong to a single region only

- $\quad$ Each region must be a connected set of pixels

- $\quad$ Each region must be uniform

- Any merged pair of adjacent regions must be non-uniform

Region growing satisfies the third and fourth of these criteria, but not the others. It fails to satisfy the first and second criteria because, in general, the number of seeds defined by the user will not be sufficient to create a region for every pixel. The fifth criterion is not satisfied because the regions grown from two nearby seeds are always regarded as distinct, even if those seeds are defined in a part of the image that should be segmented as a single region.

\subsection{Theory of Watershed Method}

Any grayscale image can be viewed as a topographic surface where high intensity denotes peaks and hills while low intensity denotes valleys. You start filling every isolated valleys (local minima) with different colored water (labels). As the water rises, depending on the peaks (gradients) nearby, water from different valleys, obviously with different colors will start to merge. To avoid that, you build barriers in the locations where water merges. You continue the work of filling water and building barriers until all the peaks are under water. Then the barriers you created gives you the segmentation result applying the watershed transform method was explained well in the results section. We used watershed transform proposed by [10]

Figure (1) shows the diagram of the processing steps of the medical images segmentation proposed system.

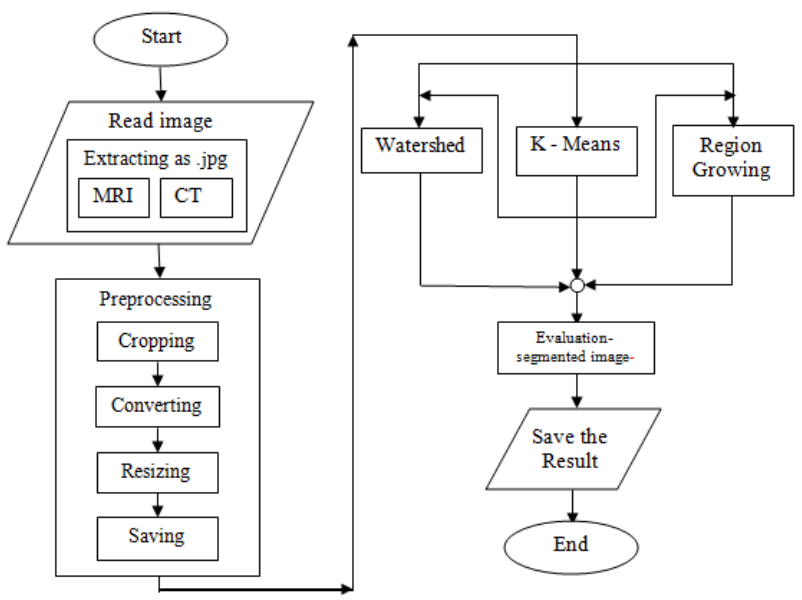

Figure 1: shows the diagram of the proposed medical images segmentation system.

\section{Results of Tested Examples}

Example 1- Spine image

After using K-means to divide the spine image into 4 regions see figure (2), we have got four values represent 4 regions as in the figure below(maximum value 216 and minimum is 32 ) the display range is $0-255$. 


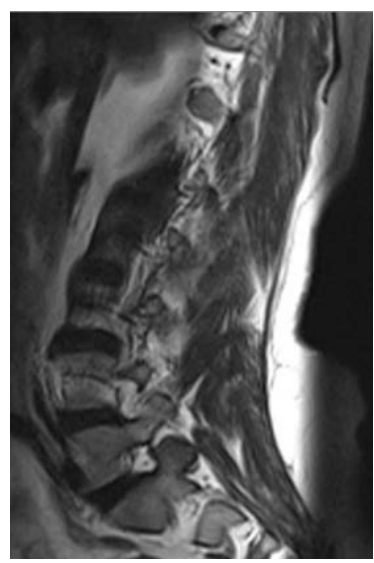

Figure 2 (a) origin image

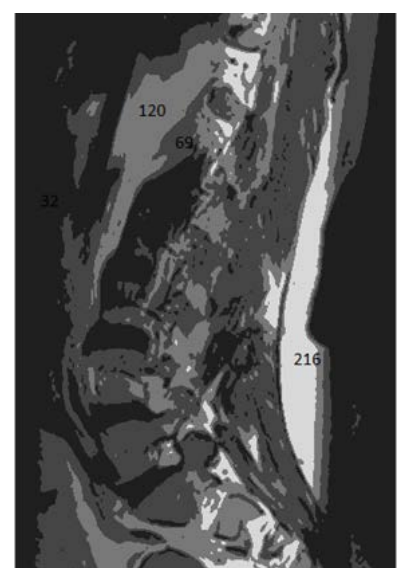

Figure 2 (b)

segmented image into 4 regions using clustering Technique (see their intensities(32, 69,120,216)

By choosing a seed point in the input image, then using region growing method to find the region boundary and the number of pixel represents this region within the threshold range depend upon the image .number of pixels is very useful to calculate the area of the effected region in the patient image before and after diagnostic by the hospital. See figure (3)

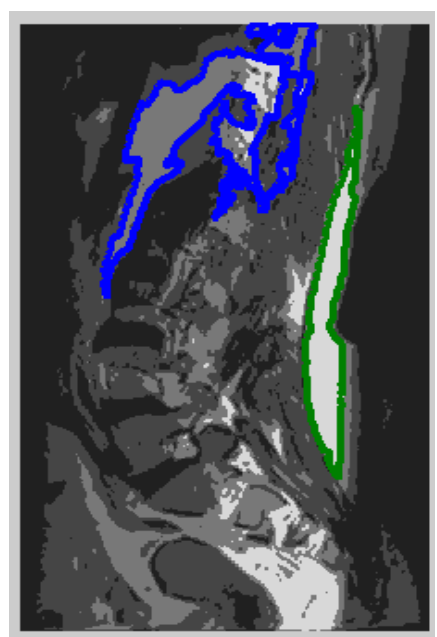

(a)

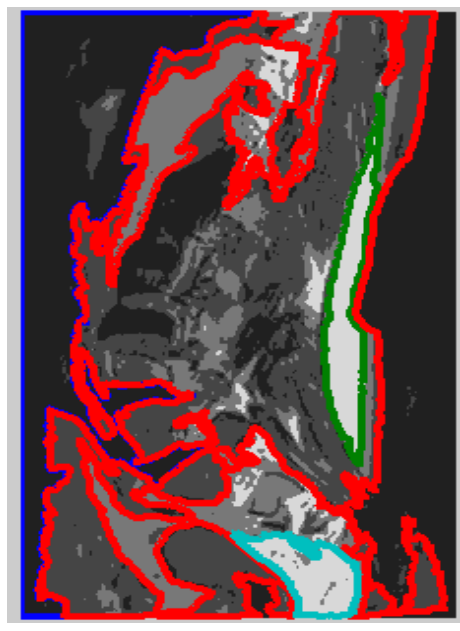

(b)

Figure 3: a) chose two regions seed and segmented(blue and green boundaries)

b) segmented image into 4 regions(red, blue, green light blue edges)

Example-2 Knee image

Using the region growing algorithm to choose the medical image (MRI, or CT, Jpeg or .mat formats) that we want to segment. inter the region number, then click somewhere inside the medical image( initial position), the region growing algorithm (regionGrowing1) was called as below, where $\mathrm{Bn}$ is the input image, and the threshold value is 2500 ( using .mat image file format here)

Poly = regionGrowing1 (Bn, [], 2500);

The output as follows, see figure (4), where the algorithm determines the initial position of seed point then calculate number of pixel represent the segmented region. 


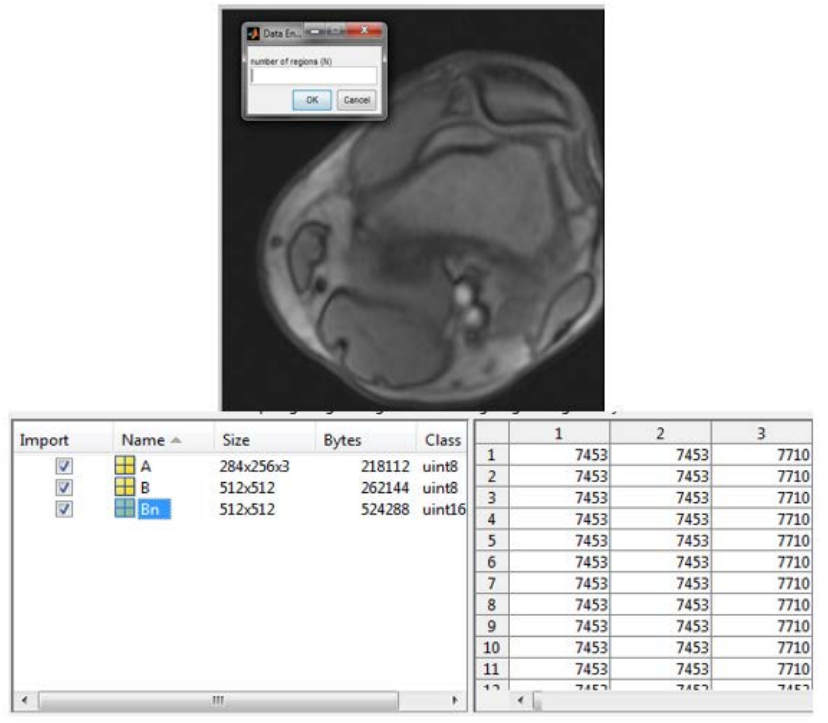

Figure 4: show the input knee image with its information as a .mat file

The segmentation result as follow, see figure (5).
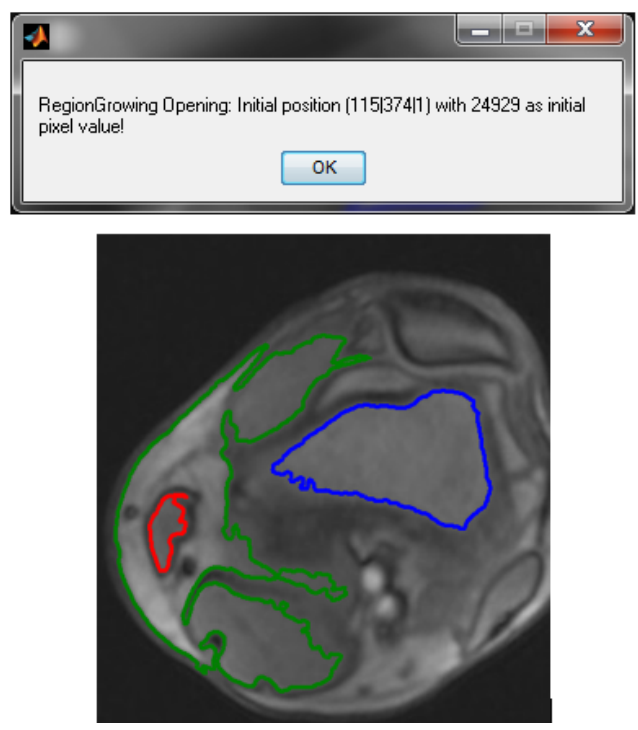

Figure 5: shows three segmented regions of origin image in figure(4)

We choose just three regions here, also there are many message box to show you our result in details. for 4 segmented region see the following figure(6).

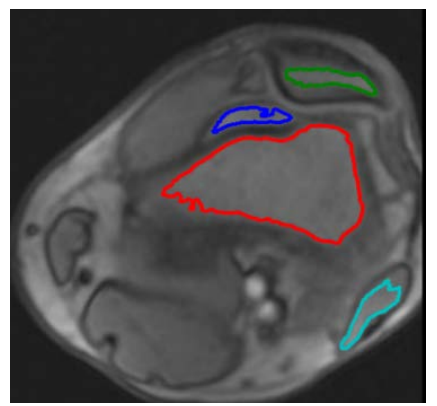

Figure 6: shows 4 segmented regions of figure(4) 
Another test for another choosing region in the same image as below.

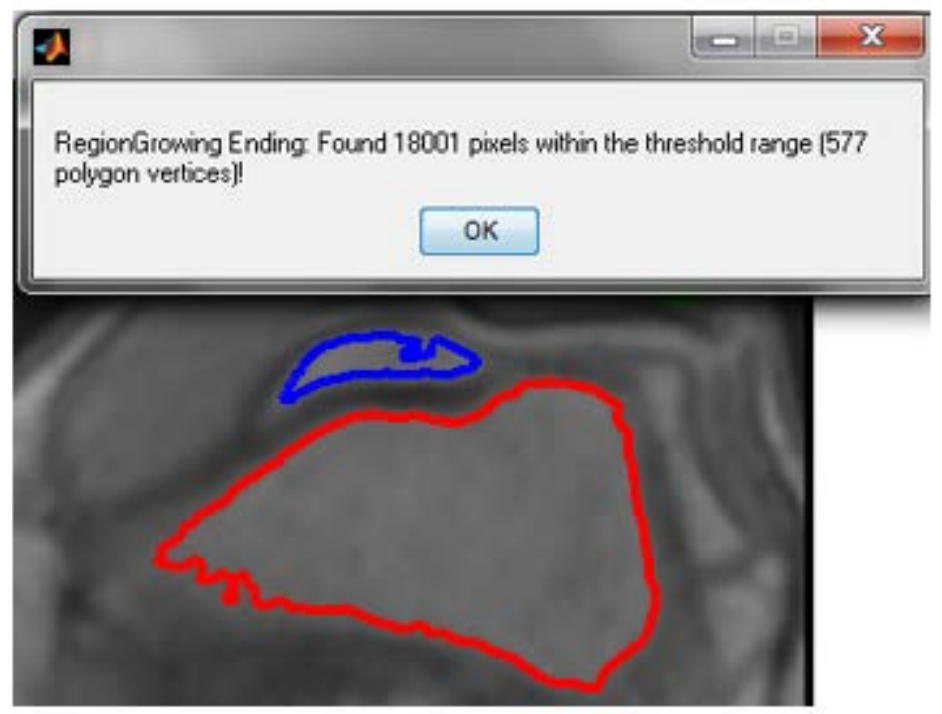

From these results we can calculate:

- $\quad$ area in pixel for effected region interested region

- $\quad$ after take medicine and take new image ,we can calculate the affected area again and compare it with first one, to see the effect of medicine or if no difference we can say the medicine is not correct.

- we can evaluate how many days the patient can be good health

- we can color the regions with their edge

here we use .mat format with uint16 format for fast processing using Matlab

Example 3-Abdomen image

Another test image, 5 regions are chosen just for example to segment abdomen image as in figure(7).
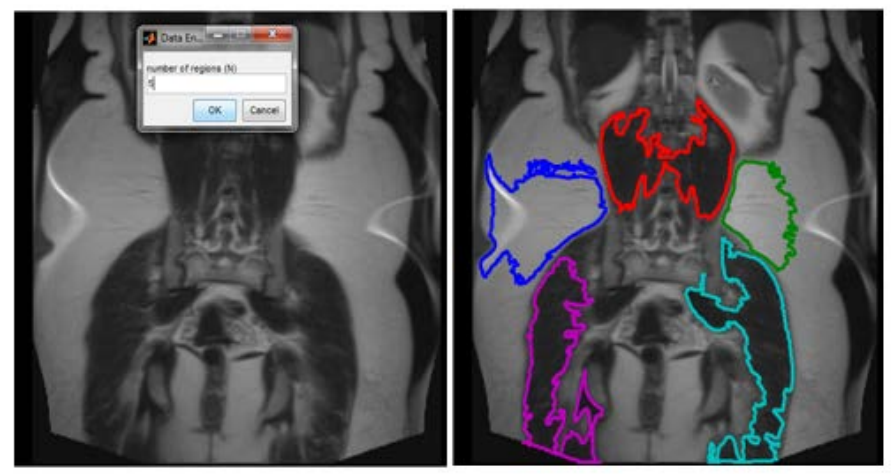

Figure 7: abdomen image segmentation into 5 chosen regions

\subsection{CT images sample using .mat and .jpg files formats}

- $\quad$ For .mat file format of input medical image,we used uint16 class, where the display range is 0-65535, it is easy to get pixel information (location + values). we used gray image , its name 002284c9.jpg from Rezqari hospital -Erbil-Iraq, it is color image 
converted into gray, resize into $512 \times 512$, save it on D:\ Bnrgray.mat to easy manipulate using Matlab

- $\quad$ For .jpg image file format

we read the original color image, then convert it into gray, also resize it into $512 \times 512$, and finally write it on $D: \backslash$ as .jpg image format

Example 4 Knee image with watershed transform

In this example watershed method was used for medical image, the result is oversegmented image see the figure (8) below.

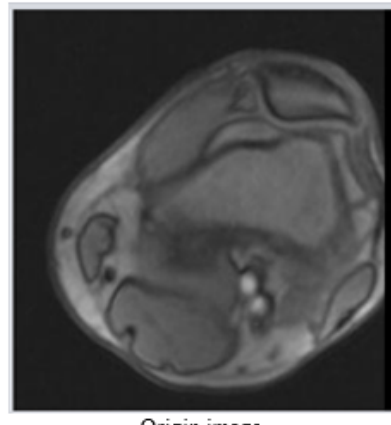

Origin image

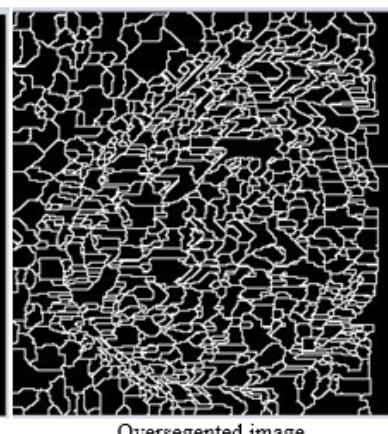

Oversegented image

Figure 8: show the over-segmentation results using watershed algorithm

The watershed transform is executed on the gradient image. This implemented by defining the first derivative of an image (measuring the change of gray levels) using $3 \times 3$ gradient operator in $x$ and $y$ directions, where the aim of the watershed transform is to search for regions of high intensity gradients (watersheds), the result is initial segmentation. With this method the marker image was used to calculate the number of pixels of each region then calculate region mean intensity value. In the merging step (on the initial segmentation image) if the difference of means value of the two regions is less than our test threshold, the two regions are merged. also we used two threshold values $\mathrm{T} 1, \mathrm{~T} 2$, and the edge strength value compare with these values as follows: , if it is for example the edge strength less than $\mathrm{T} 1$, the two adjacent regions are merged and the region becomes large(growing), and the same for $\mathrm{T} 2$.

From above we can say shortly: 1st we get merging image using mean values (initial segmentation), then using $\mathrm{T} 1$ on the mean value merging image to get new merge image. Finally the output again used with $\mathrm{T} 2$ to get the final merging region (final segmented image). The results are shown in the figure (9). 

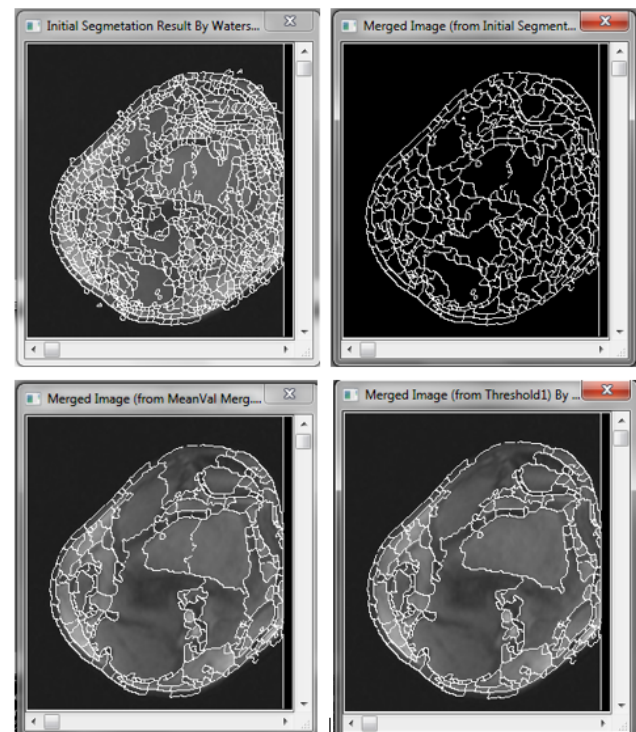

Figure(9) watershed algorithm result using mean value and two thresholding values for merging

We found that threshold 1 and threshold 2 values between $0-5$ (i.e in our case above $T 1=T 2=4$ ) give us good results, this because the nature of the image got it from Rezgari hospital, see the figure below. i.e we do simple processing on image 002284C9.jpg and so on for all other medical images. The properties of the medical image is shown in the figure below.

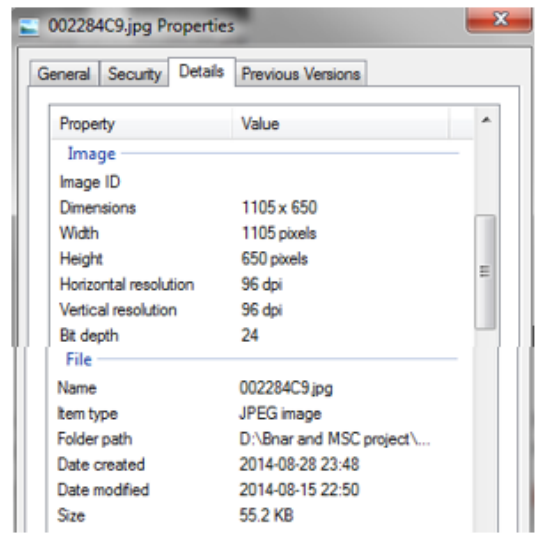

\section{Conclusions}

Applying our automated proposed segmentation system on MRI and CT images give the following conclusions:

- It is easy to read and process the .mat file and .jpg or other file formats for medical images (i.e., CT, MRI).

- $\quad$ The program determines the number of pixels of segmented area which is very important for medical image analysis for diseases or medicine effects for affected area of human body.

- $\quad$ The affected area (i.e.; region of the interest) with their edge can be colored and segmented very well with correct edge positions of each region.

- Using K-means clustering method output to region growing as input, gives accurate and very good results compare with watershed technique which depends on gradient of input image, the mean and the threshold values. 
- The manual selection of the threshold value for the watershed is not as good as automatically selecting, data misses may be happen

- $\quad$ Images can be taken in a period; before and after receiving a medicine; so after taking medicine and taking new image, the affected area can be calculated again and compare with the first one, to see the effect of medicine or if no difference appears it can be concluded the medicine did not match the disease, so the medicine is not correct.

- $\quad$ Evaluate during how many days the patients can acquire their health.

\section{REFERENCES}

[1] Neeraj Sharma and Lalit M. Aggarwal,.2010:Automated medical image segmentation techniques, Journal of Medical Physics / Association of Medical Physicists of India. Jan-Mar 2010; 35(1)3-14.

[2] Gonzalez RC, Woods RE. Digital image processing. 2nd ed. 2004. Pearson Education.

[3] Pratt KW. Digital image processing. 3rd ed. Willey; 2001. pp. 551-87.

[4] Pal NR, Pal SH. A review on image segmentation techniques. Pattern Recog. 1993;26:127794.

[5] Song T, Gasparovicc C, Andreasen N. A hybrid tissue segmentation approach for brain MR images. Med Biol Eng Comput. 2006;44:242-9.

[6] Liao L, Lin T, Li B. MRI brain image segmentation and bias field correction based on fast spatially constrained kernel clustering approach. Pattern Recog Lett. 2008;29:1580-8.

[7] Kuo WF, Lin CY, Yung-Nien Sun YN. Brain MR images segmentation using statistical ratio: Mapping between watershed and competitive Hopfield clustering network algorithms. Comput Met Prog Biomed.2008;9:191-8.

[8] Cuadra MB, Craene MD, Duay V. Dense deformation field estimation for Atlas-based segmentation of pathological MR brain images. Comput Met Prog Biomed. 2006;84:67-75.

[9] Danial kellner :Recursive region growing algorithm for 2D/3D grayscale images with polygon and binary mask output (2011). Mathwork.

[10] L. Vincent, P. Soille. Watersheds in digital spaces: An efficient algorithm based on immersion simulations [J]. IEEE Transactions on Pattern Analysis and Machine Intelligence, 1991 , 13(6): 583-593. 